\title{
IMPLANT SURGERY AND ORAL
}

ANTICOAGULANT THERAPY: CASE REPORT

\author{
M. MIRANDA ${ }^{1}$, P. BOLLERO ${ }^{2}$, N. D’OVIDIO ${ }^{1}$, V. MARSANGO ${ }^{1}$, A. BARLATTANI Jr ${ }^{1}$
}

\author{
${ }^{1}$ Department of Clinical Sciences and Translational Medicine, University of Rome "Tor Vergata”, Rome, Italy \\ ${ }^{2}$ Department of Systems Medicine, University of Rome "Tor Vergata”, Rome, Italy
}

\begin{abstract}
SUMMARY
Objectives. This work aims to assess the risks both thromboembolic that bleeding of a management protocol "non-conservative" in patients on oral anticoagulant therapy (OAT) to be undergoing implant surgery.

Materials and methods. We decided to take a surgical "non-conservative" protocol, to insert four implants in the aesthetic zone, without using flapless surgery and the surgical template. In accordance with the hematologist, the value of INR is lowered and warfarin was replaced with heparin low molecular weight, to have a better coagulation's control.

Results. The modern guidelines impose a protocol of conservative management in patients with OAT, with minimally invasive surgery, flapless, and use of surgical template to reduce the risk of uncontrolled bleeding. This, thanks to the teamwork between dentist and hematologist, thanks to careful adjustment of INR and the use of local haemostatic agents, were not encountered any problems with bleeding or intra or postoperative.

Conclusion. Surgical treatment of patients with OAT is a real problem for the oral surgeon, to treat every time in association with the hematologist. Applying this type of surgical procedure, different from today's guidelines, in our experience there were no post-operative complications (bleeding or bleeding); osseointegration has not been compromised and the prosthetic rehabilitation was completed successfully.
\end{abstract}

Key words: oral pathology, implant surgery, OAT, protocol "non conservative".

\section{Introduction}

The number of patients in dental surgical oral anticoagulant treatment is increasing and this is mainly due to the continuous increase of cardiovascular disease. The OAT is the golden goal for the treatment and prevention of thromboembolic disease and vascular type in general, such as in carriers of prosthetic heart valves $(1,2)$ or valvular heart disease, in case of cardiac thrombosis intracavitary, atrial fibrillation (3-6), atrial flutter (7), acute myocardial infarction (8), dilated cardiomyopathy (9), ischemic stroke (10), peripheral arterial disease (11), prevention of postoperative thromboembolism (12), treatment of VTE and prevention of relapse (13), and antiphospholipid syndrome $(14,15)$. While patients with normal clotting parameters, have an INR between $0.7-1.2$, the patients in TAO generally have val- ues between 2 and 4, that indicate a more blood's fluidity. The risk of thrombosis is increased by a decrease of the normal range. Before proceeding, we have to assess the thromboembolic risk related to the disease, and bleeding risks related to surgery.

The treatments with bleeding's risk include the use of injectable anesthetics, tooth extractions, placing implants osseointegrated, periodontal surgery and periodontal curettage, minor surgery and root canal therapy $(16,17)$. The knowledge of the correct protocol outpatient management, is a prerequisite for starting treatment. To date, however, the management of these patients for minor oral surgery (such as the osseointegrated implants) is still a complex issue, with a strong debate between the risk of uncontrolled bleeding in case of no discontinuation of therapy or the possibility of thromboembolic complications in the case of suspension of the medication (18). In 


\section{case report}

this case report, we want show that it was possible the insertion of 4 plants, one of which is post-extractive without the occurrence of postoperative bleeding complications, thromboembolic or bruising, with a non-flapless surgery and without use of the drilling template. Thanks to the teamwork between dentist and hematologist, the value of INR is lowered at 1.7, and warfarin has been replaced with heparin low molecular weight. The most important things, before any oral surgery, is individualized a correct management protocol. Targeted's protocol is necessary in high tromboembolic risk patients to prevent a thromboembolic event, at the expense of a slightly increased risk of bleeding especially in cases of minor oral surgery (18).

\section{Materials and methods}

At the Department of Special Pathology Odontostomatology of the "Policlinico Tor Vergata", we treated a 68-year-old patient, sex M, with a history of atrial fibrillation, in treatment with warfarin and Almarythm. The RX orthopanoramic evidents edentulous areas corresponding to the elements $1.1,1.2,2.1,2.2,2.3$ and the presence of residual root of 1.3 with permanent rehabilitation gold-porcelain-type "full-arch". At the last blood test, the value of INR is 3 .

The fixed prosthetic restoration is fractured and there are carious lesions in the cervical third load of 1.3 (Figs. 1, 2).

We decided to rehabilitate the anterior area by placing 4 implants in the position 1.2, 1.3, 2.2 and 2.3. In agreement with the hematologist, we suspend the assumption of warfarin and we started the therapy with heparin low molecular weight to correct altered coagulation's values.

The INR at the time of surgery is 1,7. Heparin was discontinued 10 hours before the surgical intervention. In addition, the two days before the surgery, the patient started antibiotic prophylaxis with $1 \mathrm{~g}$ of amoxicillin and clavulanic acid every 12 hours. Before starting the surgery we gave a rinse of 60 sec. with chlorhexidine digluconate $0.2 \%$. The two days before surgery, the patient started an- tibiotic prophylaxis with $1 \mathrm{gr}$ of amoxicillin and clavulanate every 12 hours.

The surgery begins with preliminary anesthesia with articaine 1/200.000 making a slow infusion. We did the extraction of the residual root of 1.3, followed by thorough bone's toilet and removal of any granulation tissues bleed easily (Fig. 3).

We did crestal incision, near the palatal side, without flap-less surgery and surgical template (to reduce time and costs of labor) (Fig. 4).

We did the mechanical preparation, using Winsix's incremental pre-calibrated drills of length $13 \mathrm{~mm}$, maintaining an under-preparation of 2 $\mathrm{mm}$ in each implant's locus, due to the compactness of bone type D2. We insert a post-extraction implant Winsix TTx Micro Rough Surface internal hex conical, lenght $13 \mathrm{~mm}$ and diameter 4.5 $\mathrm{mm}$ in area 1.3 , inserting the vestibular cortical

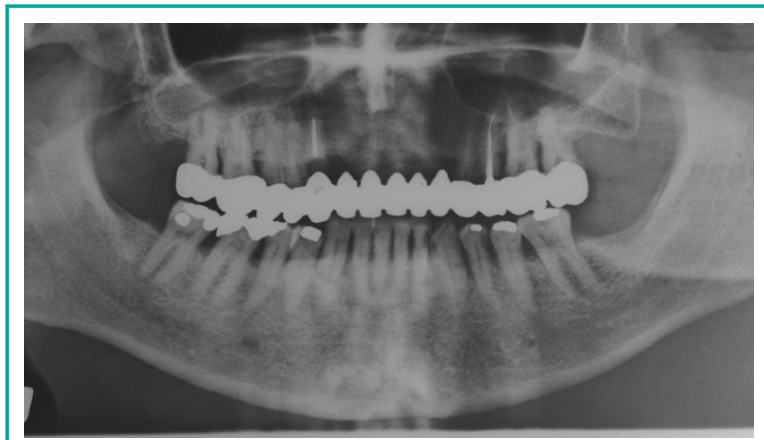

Figure 1

Rx Orthopanoramic before treatment.

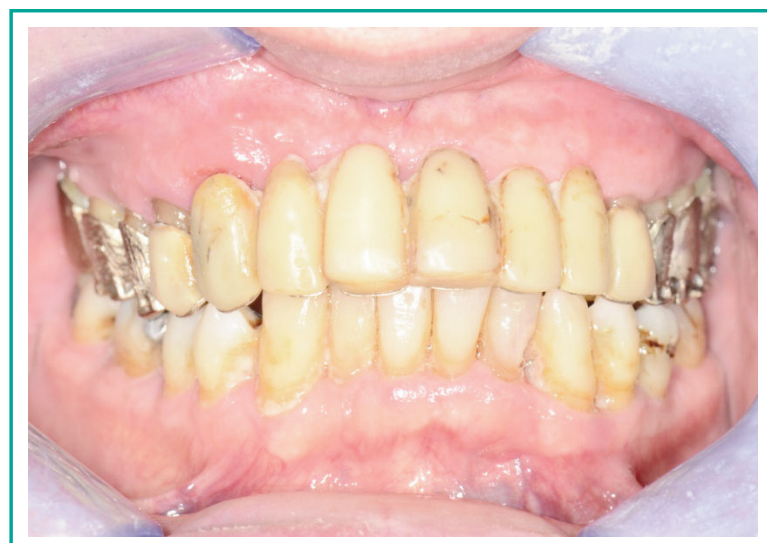

Figure 2

Intraoral view before treatment. 


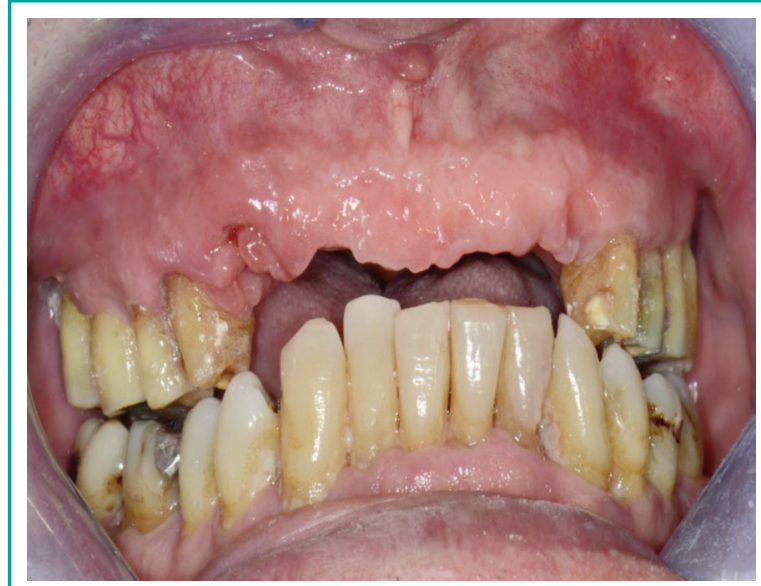

Figure 3

The maxilla after extraction of residual root 1.3.

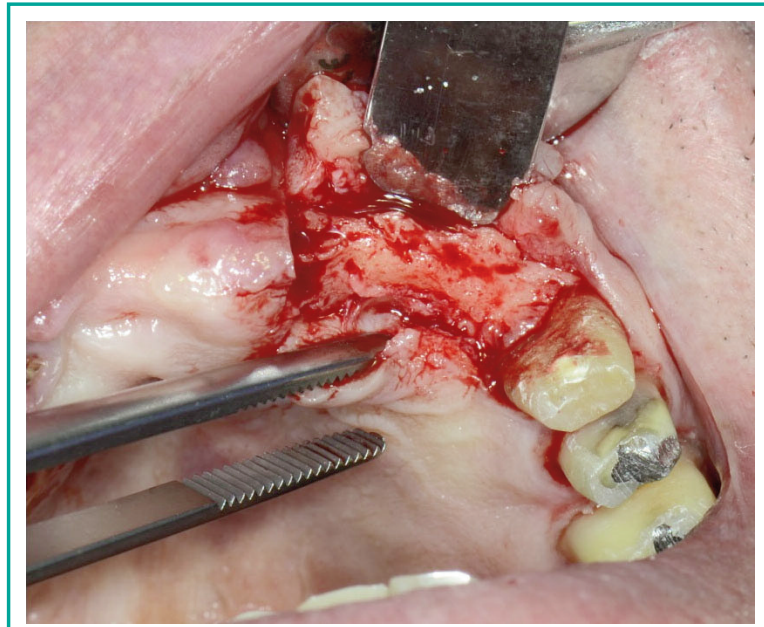

Figure 4

Crestal incision before the implants' insertion.

granules obtained by milling. In the other areas we opted for implants Winsix TTx $13 \mathrm{~mm}$ diameter and $3.8 \mathrm{~mm}$, placing the screw cap Winsix and sutured with Vycril 3/0 (Fig. 5).

We irrigated the surgical field with tranexamic acid solution and applied where necessary, fibrin glue. After 30 minutes, the bleeding was completely absent. Because it was the aesthetic zone, we proceed to the reposition of prosthetic restoration, previously modified, using the stumps remaining 1.5, 2.4 and 2.5. After 10 hours of surgery, the heparin is administered again, and then suspended entirely, when the
INR values were higher than 2.0 for two times, as suggested by Rossi \& Magrella (19). After two samples with an INR $>2$, the warfarin therapy was taken up only.

The timing phasing is maintained until the complete osseointegration of the implant fixture. We decided to maintain on-site restoration in the esthetic zone, for 4 months. The patient decided, for economic issues, to finalize the work with elements in reinforced resin, rather than ceramic (Figs. 6, 7, 8).

The discharge of the patient is not made before 60 minutes of the end of the surgery (to verify the absence of bleeding) and we proceeded to the application of an ice pack for 3-4 hours. For the 3 days after surgery, patient followed a liquid diet

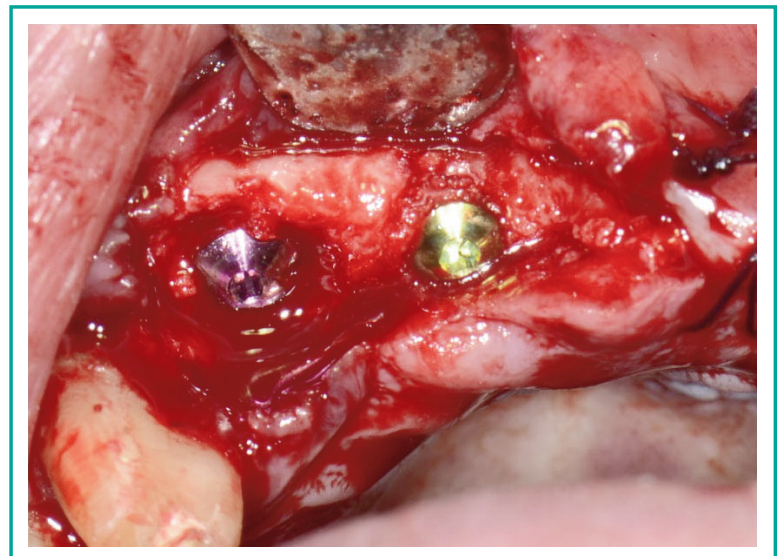

Figure 5

Winsix implants and screws cap in position 1.3 and 1.4.

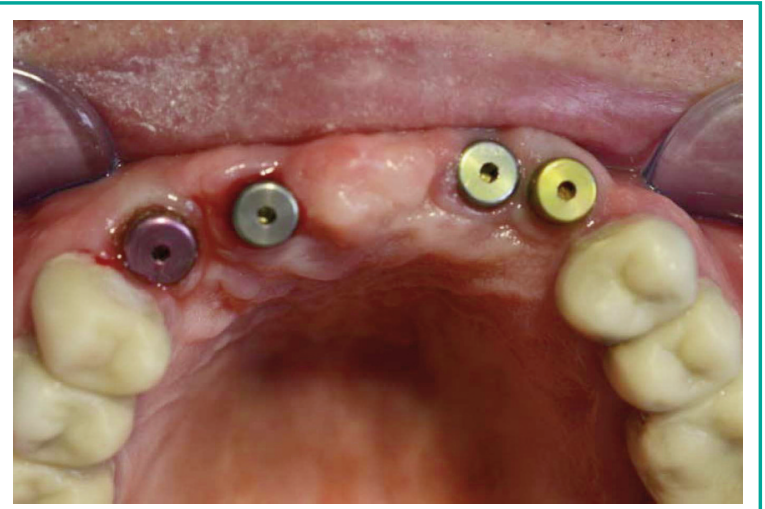

Figure 6

After 4 months the placement of the healing screws. 


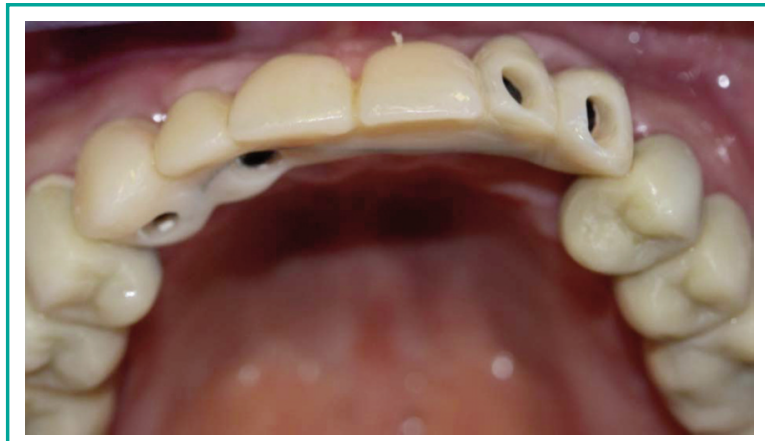

Figure 7

The provisional elements in reinforced resin before their fixing with screws.

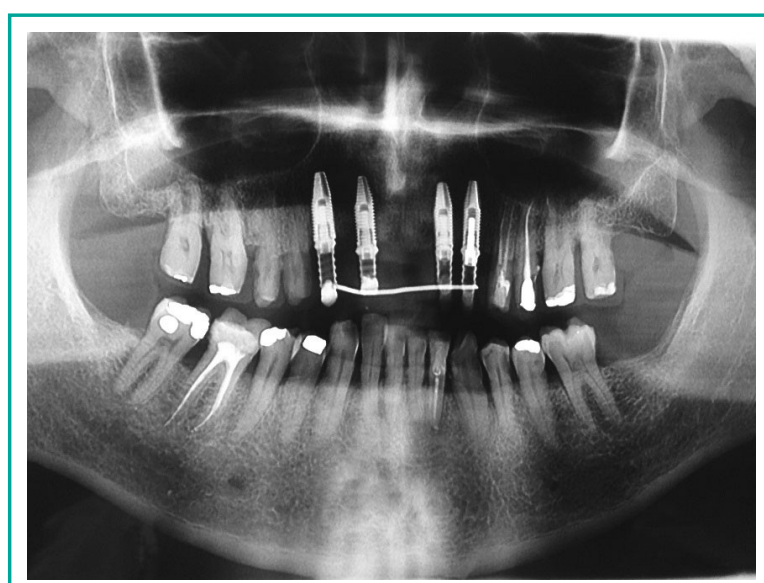

Figure 8

Rx Orthopanoramic after treatment.

and cold for the 4 following weeks, soft and warm. We prescribed oral rinse with $10 \mathrm{~mL}$ of aqueous solution of tranexamic acid for 2 minutes, repeated 4 times a day for 7 days. He couldn't eat or drink for about an hour after rinsing and oral rinses with chlorhexidine digluconate $0.12 \%$ twice a day (morning and evening) as suggested by Gonty (20). The anti-inflammatory therapy if required, was based on the use of paracetamol.

\section{Results}

All patients should be directed to their hematologist for adjustment of therapy, before any inva- sive dental procedure. Applying this type of "non-conservative" protocol, associated with the adjustment of INR, there were no post-operative complications in our case (uncontrolled bleeding or thromboembolic phenomenon).

Osseointegration was minimally impaired and prosthetic rehabilitation was completed successfully.

\section{Discussion}

For the dental treatment of patients with OAT, is important to value the surgical risk and the possibility of thromboembolism and, it is important to perform a careful history and thorough evaluation of INR (21). Garcia et al. in 2008 published an important prospective study on the risk of thromboembolism after a brief suspension of the OAT, reporting an incidence of thromboembolic events of $0.5 \%$, in a follow-up period of 30 days (22). In the same study is also emphasized as the majority of postoperative bleeding begins at a distance of 2-3 days after surgery, by the action of plasminogen activators, resulting in clot lysis (crankcase). The surgery and local anesthesia should be performed in the least traumatic way, otherwise it would cause massive bruising. With regard to the guidelines in oral surgery in patients with OAT, the review by the American College of Chest Physicians recommends always co-administration of a pro-haemostatic local agent (23). Guidelines doesn't do differences between big or small surgery. In agreement with Scully et al. (2007), the trauma for the positioning of a plant, is the same of the extraction of 3 teeth, provided that these processes do not involve collection of autologous bone grafts, the execution of large flaps or the placement of installations in sites where there is a risk during the preparation of the osteotomy to extend outside of the bone marrow (24). The reduction in the perioperative period of warfarin dosing or discontinuation of anticoagulant treatment 2 or 3 days before oral surgery procedure is a strategy widely used. It aims to decrease the value of INR with the pre- 
diction that in the next $48-72$ hours, will be in the range 1.5-2.1 (25). The complete suspension of the OAT is often recommended to reduce the risk of post-operative bleeding, resulting in 4 days the return to a state of coagulation of a typical normal subject. This strategy may, however, expose the patient to increased risk of thromboembolic events (26). The need to prevent an attack of thromboembolism, in patients, classified as high risk of thromboembolism, should dictate the use of a Bridging Therapy's protocol for the perioperative management, regardless of the risk of bleeding, especially in cases of minor oral surgery.

It's been shown from Madrid and Sanz in 2009 that patients on OAT (with INR between 2 and 4) who do not suspend the drug, do not present an increased risk of postoperative bleeding compared with patients who discontinue oral application; also the application of local hemostatic agents is very effective to prevent postoperative bleeding. The comparison carried out by the authors, among the various hemostatic agents (rinses with tranexamic acid, gelatin sponges or fibrin glue) showed a similar positive effect in the results (18).

\section{Conclusions}

Surgical treatment of patients with OAT is a real problem for the oral surgeon. Although there is a theoretical risk of hemorrhage following surgery, using our protocol based on the suspension of warfarin and the beginning of heparin, the risk of bleeding appears to be minimal and easily controlled by the use of local hemostatic.

This risk seems to be minimal and the bleeding controllable through the use of local measures of hemostasis. This risk is, however, much less important than the risk of thromboembolism associated with the discontinuation of therapy (27). Based on the above, we can therefore say that the suspension of OAT is not recommended in cases of minor oral surgery, such as a single tooth extraction, predisposing the subject to greater risks than benefits. The application of lo- cal hemostatic agents is always recommended after each surgery (e.g. tranexamic acid 4.8\% 10 $\mathrm{ml}, 4$ times/day for 2 days), gelatin sponges containing thrombin or biological adhesives: all with the same effective power in the control of postoperative bleeding. We can also conclude that despite the modern management recommending a "conservative" protocols of the patient, in our case we have adopted a "normal" management protocol, so without using flapless surgery and surgical template, and found no bleeding or thromboembolic phenomena in the post-operative thanks to the careful preliminary assessment and the teamwork between dentist and hematologist. In addition, we can reduce the timing of work, as well as costs of treatment, without the use of surgery flapless and/or surgical template. The placement of osseointegrated implants is not absolutely contraindicated in patients taking anticoagulants, but always we adopt an appropriate management protocol in accordance with the modern guidelines.

\section{References}

1. Acar J, Iung B, Boissel JP, et al. AREVA: multicenter randomized comparison of low-dose versus standarddose anticoagulation in patients with mechanical prosthetic heart valves. Circulation. 1996;94:2107-12.

2. Salem DN, Stein PD, Al-Ahmad A, et al. Antitrombotic therapy in valvular heart disease-native and prosthetic: the Seventh ACCP Conference on Antithrombotic and Trombolytic Therapy. Chest. 2004;126:S457-82.

3. Singer DE, Albers GW, Dalen JE, et al. Antithrombotic therapy in atrial fibrillation: the Seventh ACCP Conference on Antithrombotic and Thrombolytic Therapy. Chest. 2004;126:S457-82.

4. Atrial fibrillation investigators. Risk factors for stroke and efficacy of antithrombotic therapy in atrial fibrillation. Analysis of pooled data from five randomized controlled trials. Arch Intern Med. 1994;154:1449-57.

5. Waldo AL, Becker RC, Tapson VF, et al. Hospitalized patients with atrial fibrillation and a high risk of stroke are not being provided with adequate anticoagulation. $\mathrm{J}$ Am Coll Cardiol. 2005;46:1729-36.

6. Miyasaka Y, Nbarnes ME, Gersh BJ, et al. Secular trends in incidence of atrial fibrillation in Olmstead County, Minnesota,1980-2000, and implications on the projection for future prevalence. Circulation. 2006; 114:970-9. 
18. Madrid C, Sanz M. What influence do anticoagulants have on oral implant therapy? A systematic review. Clin Oral Impl Res. 2009;20:96-106.

19. Rossi F, Magrella M. I farmaci del sangue. Basi farmacologiche della medicina. Vol IV. Torino:UTET;1997.

20. Gonty AA. Dental extractions in patient on an oral anticoagulant: a survey of practices in North America. J Oral Maxillofac Surg. 1998;56:917.

21. Meehan S, Schmidt MC, Mitchell PF. The International Normalized ratio as a measure of anticoagulation: significance for the management of the dental outpatient. Spec Care Dent. 1997;17(3):94-6.

22. Garcia DA, Regan S, Henault LE, Upadhyay A, Baker J, Othman M, Hylek EM. Risk of thromboembolism with short-term interruption of warfarin therapy. Archives of Internal Medicine. 2008;168:63-69.

23. Douketis JD, Berger PB, Dunn AS, Jaffer AK, Spyropoulos AC, Becker RC, Ansell J. American College of Chest Physicians. The perioperative management of antithrombotic therapy: American College of Chest Physicians Evidence-Based Clinical Practice Guidelines (8th Edition). Chest. 2008;133:299S-339S.

24. Scully C, Hobkirk J, Dios PD. Dental endoosseus implants in the medically compromised patient. Journal of Oral Rehabilitation. 2007;34;590-99.

25. Mulligan R, Weitzel KG. Pretreatment management of the patient receiving anticoagulant drugs. Journal of the American Dental Association. 1988;117:479-483.

26. Dunn AS, Turpie AG. Perioperative management of patients receiving oral anticoagulants: a systematic review. Archives of Internal Medicine. 2003;163:901-908.

27. Wahl MJ. Demystifying medical complexities. Journal of the Californian Dental Association. 2000;28:510-518.

Correspondence to:

Michele Miranda, MD

Department of Clinical Sciences and Translational Medicine

University of Rome "Tor Vergata"

Via Montpellier 1, 00133 Rome, Italy

E-mail: michelemiranda.ptv@gmail.com 